\title{
Diode laser operation under orthogonal feedback: experiments and theory
}

\author{
Taciano Sorrentino \\ Campus Caraúbas \\ Universidade Federal Rural \\ do Semi-Árido \\ Caraúbas, Brazil \\ e-mail: taciano@ufersa.edu.br
}

\author{
Cristina Masoller \\ Departament de Fisica i Enginyeria Nuclear \\ Universitat Politècnica de Catalunya \\ Terrassa, Spain \\ e-mail: cristina.masoller@upc.edu
}

\author{
César Soares, Itamar Vidal and Marcos Oriá \\ Laboratório de Física Atômica e Lasers, \\ Departamento de Física \\ Universidade Federal da Paraíba \\ João Pessoa, Brazil \\ e-mail: oria@otica.ufpb.br
}

\begin{abstract}
We report experimental and numerical investigations concerning semiconductor lasers emission under polarization-rotated feedback. We experimentally observe that for some semiconductor lasers the weak TM mode does lase even in the absence of TM feedback and we monitor how the TE and TM emissions evolve with the increase of the TM feedback strength. The emission frequency of a semiconductor laser subject to polarization-rotated feedback experiences a shift proportional to the optical power fed back into the laser [1]. We aim to investigate what affects the sensibility of the frequency of semiconductor lasers to polarization-rotated feedback. Experimentally, we measured the proportionality coefficient $\beta$ between frequency shift and feedback power for different lasers and characterized how it varies with some parameters. Theoretically, we made numerical calculations using a rate equation model [2], and our results show a critical dependence of the $\beta$ coefficient on the gain self- and cross-saturation of both modes and the relations between these saturation coefficients.
\end{abstract}

\section{INTRODUCTION}

Orthogonal optical feedback has been used in semiconductor lasers either to improve frequency stability and spectral characteristics [3], [4], or to induce instabilities and dynamical behavior [1], [5], [6], and some other applications [7], [8], [9], [10]. Since the first report by Kawaguchi in 1988 [3], some of these applications take advantage of the emission frequency dependence on the orthogonal feedback power. This dependence was shown to be linear [1], but what makes the semiconductor laser frequency more or less influenced by the feedback power is a question still not addressed. The aim of this work is to investigate, experimentally and numerically, the parameters that affect the sensibility of the laser frequency to the orthogonal feedback. Furthermore, we found that for some of the lasers we investigated in the experiments the weak TM mode emission shows laser characteristics, even without feedback. This observation differs from those in other reports where the TM mode is found to oscillate only in the presence of TM feedback [11], or where its found to not oscillate at all [12]. The TM oscillation is taken into account for obtaining the numerical results we present here.

\section{EXPERIMENTAL SETUP}

The experimental setup is depicted in Fig. 1. A monomode, Fabry-Prot cavity, diode laser (DL) is current and temperature

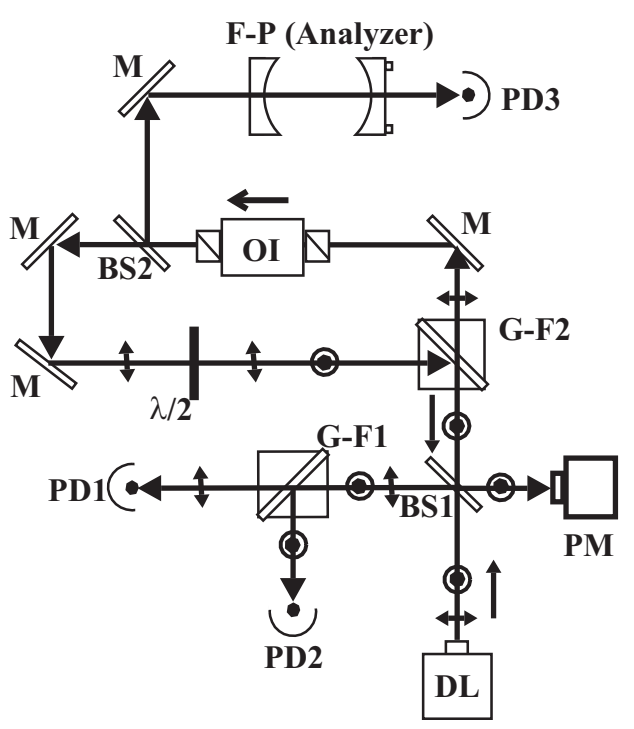

Fig. 1. Experimental setup. DL: diode laser; BS: beamsplitter; PM: powermeter; G-F: Glan-Foucault polarizer; OI: optical isolator; M: mirror; F-P: Fabry-Pérot interferometer; PD: photodetector; $\lambda / 2$ : half-wave plate.

stabilized within $10^{-4}$. The laser output is composed mainly by a TE polarization mode (oscillation in the plane of the semiconductor junction, double arrow symbol in Fig. 1), with a small part of TM-polarized light (circle with point symbol). The power ratio between these two components of polarization varies from laser to laser, and even for the same model the power ratios can be very different. We measured TE to TM intensity ratio between about one hundred and more than one thousand.

The laser emission is sent thought a beamsplitter (BS1) that deflects a fraction of its power to a Glan-Foucault polarizer (G-F1) (rejection level of $55 \mathrm{~dB}$ ) where the polarization components are separated and sent to photodetectors. The intensities of the TE and TM components are monitored by photodetectors PD1 and PD2, respectively. The beam going through BS1 is sent back to the laser in a feedback loop. A second Glan-Foucault polarizer (G-F2) filters the TE polarization and allows only TM field to be re-injected. 


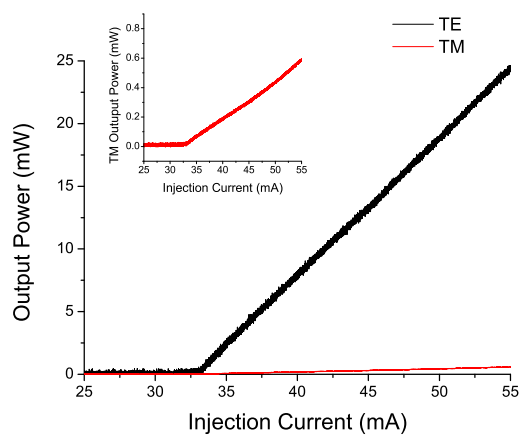

Fig. 2. TE (black) and TM (red) P-I curves without feedback for a Sanyo 7140 diode laser, with $P_{T E} / P_{T M}=53$. Inset: Detail of the TM power showing the laser threshold about $33 \mathrm{~mA}$.

The small TM-polarized reflection from the beam entering the feedback loop is used for the judicious alignment of the feedback beam leaving the feedback loop. A beamsplitter (BS2) in the feedback loop sends a small part of the beam to a Fabry-Pérot interferometer (F-P) that allows the analysis of the feedback-induced frequency shifts. An optical isolator assures the one-way character of the feedback loop and avoids TE feedback from the Fabry-Pérot cavity. A half-wave plate $(\lambda / 2)$ allows the control of the feedback power. In its way back to the laser cavity, part of the TM feedback field is sent to a powermeter (PM) by the first beamsplitter (BS1), for monitoring the feedback power.

\section{EXPERIMENTAL RESULTS}

Although various previous works in the literature reported that the TM mode oscillates only in the presence of polarization-rotated feedback [11] and even that this mode does not oscillate at all [12], we found that for some diode lasers the TM-mode emission shows laser characteristics, even in the absence of feedback. The polarization-resolved P-I curves for one of these diode lasers (Sanyo DL 7140-201S), without feedback, are shown in Fig. 2. The threshold current is the same for both polarizations. For the lasers we analyzed, we found that both polarization components have the same wavelength and very similar linewidths. We remark that the authors of reference [13] also observed that for the lasers they used the optical spectra of TE and TM modes are identical up to the resolution limit of their wavemeter.

The measured powers of the TE and TM emissions as functions of the TM feedback power, for (two different) Sanyo DL 7140 diode lasers in the same operation conditions, are shown in Fig. 3 and Fig. 4, respectively. As the TM feedback power increases, the TE output power decreases and the TM output power increases. This behavior can be explained in a simple way: as the number of TM-polarized photons in the cavity increases, more carriers recombine generating more TM-polarized photons and the carrier density contributing to the TE field is depleted. The saturation behavior in Fig. 4 was also observed for other lasers and is under investigation. A more detailed description will be presented elsewhere.

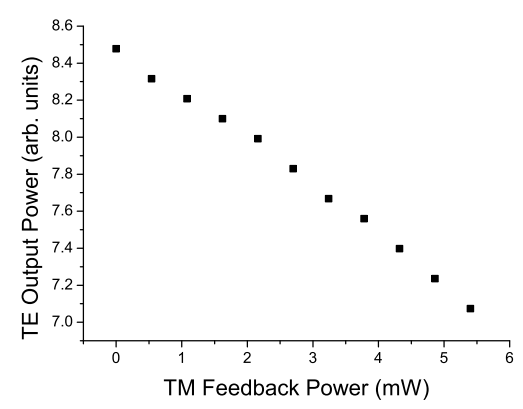

Fig. 3. TE output power as a function of TM feedback, for a Sanyo 7140 diode laser. Injection current: $90 \mathrm{~mA} . P_{T E} / P_{T M}=338$.

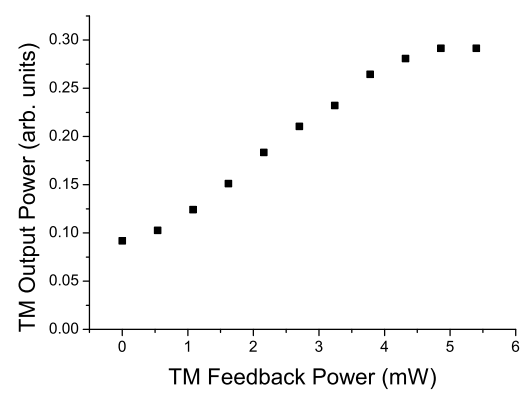

Fig. 4. TM output power as a function of TM feedback, for a Sanyo 7140 diode laser. Injection current: $90 \mathrm{~mA}$. $P_{T E} / P_{T M}=219$.

The emission frequency of a semiconductor laser under polarization-rotated feedback experiences a shift from its free running value that is linear with the feedback power [5], [1], [6]:

$$
\nu_{0}-\nu=\beta P_{f}
$$

The $\beta$ coefficient in equation (1) was obtained from first principles in [1], and seems to not vary with temperature or injection current. In order to investigate the factors that determine the sensibility of the emission frequency of a semiconductor laser to polarization-rotated optical feedback, we measured beta as a function of various parameters for different lasers.

Experimentally we observe that the value of $\beta$ does not vary systematically with the temperature or injection current. However, $\beta$ depends critically on the power ratio between the two orthogonal modes. Fig. 5 shows $\beta$ as function of the power ratio between the two polarizations $\left(P_{T E} / P_{T M}\right)$ for five lasers of the same model (Sanyo DL 7140) under the same operation conditions.

\section{NUMERICAL RESULTS}

We use a simplified version of the model presented in [2] to simulate the laser under orthogonal optical feedback, with the two polarization modes lasing. The anisotropies between the orthogonal modes are neglected, the differences between 


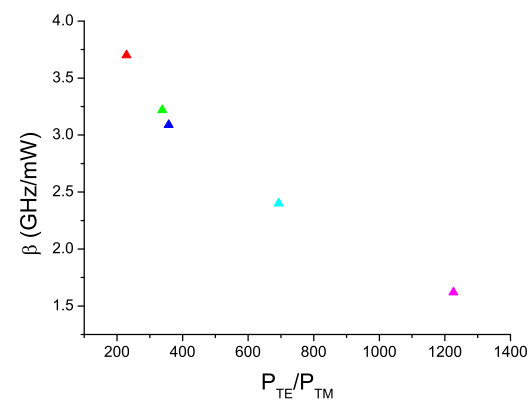

Fig. 5. $\beta$ as a function of the power ratio between TE and TM modes for five different DL 7140 diodes.

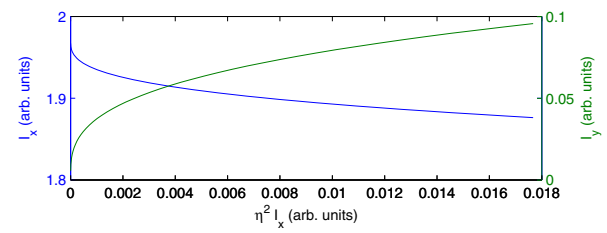

Fig. 6. TE (blue) and TM (green) intensities as functions of the feedback power for a fixed current.

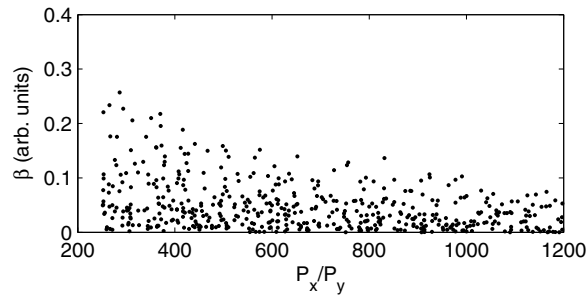

Fig. 7. $\beta$ vs. TE/TM power ratio (with no feedback) for 1000 randomly chosen values of the saturation coefficients that satisfy the experimental and stability conditions of the model.

them being the modal gains that include different self- and cross-saturation coefficients. Fig. 6 shows how the intensities of the two modes behave as the feedback power increases, resembling the experimental cases of Fig. 3 and Fig. 4. TE mode intensity decreases and TM mode intensity increases as the TM feedback power increases. Fig. 7 shows $\beta$ as a function of the TE/TM power ratio for the solitary laser, for 1000 randomly chosen values of the saturation coefficients that satisfy the experimental conditions and the stability conditions for the model. For some of these values the dependence of $\beta$ with the intensity ratio resembles the experimental case on Fig. 5. The TE/TM intensity ratio depends on the self- and cross-saturation coefficients of the two modes.

\section{CONCLUSION}

The weak TM emission of some semiconductor lasers was observed to lase without TM feedback, in contrast with some previous reports. The output power of the TE (TM) mode was experimentally observed to decrease (increase) with the increase of TM feedback. Concerning the sensibility of diode lasers frequency to orthogonal optical feedback, we observed experimentally that the proportionality coefficient between frequency shift and feedback power, $\beta$, does not vary systematically with injection current or temperature, but depends on the power ratio between the two orthogonal modes. A rate equation model used to simulate numerically the laser under orthogonal feedback allowed results in good agreement with the experimental observations and demonstrated the dependence of $\beta$ on the self- and cross-saturation coefficients of the two polarization modes.

\section{ACKNOWLEDGMENT}

C.M. is supported in part by EOARD under grant FA865510-1-3075, the ICREA ACADEMIA program and the Spanish Ministerio de Educacion y Ciencia through project FIS200913360-C03-02.

\section{REFERENCES}

[1] C. Masoller, T. Sorrentino, M. Chevrollier, and M. Oriá, "Bistability in semiconductor lasers with polarization-rotated frequency-dependent optical feedback," IEEE J. Quantum Electron., vol. 43, no. 3, pp. 261268, Mar. 2007.

[2] C. Masoller, D. Sukow, A. Gavrielides, and M. Sciamanna, "Bifurcation to square-wave switching in orthogonally delay-coupled semiconductor lasers: theory and experiment," Phys. Rev. A, vol. 84, p. 023838, 2011.

[3] H. Yasaka and H. Kawaguchi, "Linewidth reduction and optical frequency stabilization of a distributed feedback laser by incoherent optical negative feedback," Appl. Phys. Lett., vol. 53, no. 15, pp. 1360-1362, Oct. 1988

[4] A. F. A. da Rocha, P. C. S. Segundo, M. Chevrollier, and M. Oriá, "Diode laser coupled to an atomic line by incoherent optical negative feedback," Appl. Phys. Lett., vol. 84, p. 179, Jan. 2004.

[5] B. Farias, T. P. de Silans, M. Chevrollier, and M. Oriá, "Frequency bistability of a semiconductor laser under a frequency-dependent feedback," Phys. Rev. Lett., vol. 94, pp. 173 902-1, May 2005.

[6] M. Oriá, B. Farias, T. Sorrentino, and M. Chevrollier, "Multistability in the emission frequency of a semiconductor laser," J. Opt. Soc. Am. B, vol. 24, pp. 1867-1873, Aug. 2007.

[7] K. Otsuka and J. L. Chern, "High-speed picosecond pulse generation in semiconductor lasers with incoherent optical feedback," Opt. Lett., vol. 16, no. 22, pp. 1759-1761, 1991.

[8] T. C. Yen, J. W. Chang, J. M. Lin, and R. J. Chen, "High-frequency optical signal generation in a semiconductor laser by incoherent optical feedback," Opt. Commun., vol. 150, p. 158, 1998.

[9] D. L. Cheng, T. C. Yen, J. W. Chang, and J. K. Tsai, "Generation of high-speed single-wavelength optical pulses in semiconductor lasers with orthogonal-polarization optical feedback," Opt. Commun., vol. 222, p. $363,2003$.

[10] T. Sorrentino, O. Di Lorenzo, L. C. de Oliveira, M. Chevrollier, and M. Oriá, "All-optical frequency-controlled frequency switch," J. Opt. Soc. Am. B, vol. 27, no. 7, pp. 1458-1463, Jul. 2010.

[11] Y. Takeuchi, R. Shogenji, and J. Ohtsubo, "Chaos dynamics in semiconductor lasers with polarization-rotated optical feedback," Opt. Rev., vol. 17, no. 3, pp. 144-151, 2010.

[12] R. Ju and P. S. Spencer, "Dynamic regimes in semiconductor lasers subject to incoherent optical feedback," J. Lightw. Tech., vol. 23, no. 8, pp. 2513-2523, Aug. 2005.

[13] L. Khaykovich, T. Galfsky, Z. Shotan, and N. Gross, "Te-tm coupled mode dynamics in a semiconductor laser subject to feedback with variably rotated polarization," Opt. Commun., vol. 282, pp. 2059-2061, 2009. 